\title{
Interactive comment on "Improvement in tropospheric moisture retrievals from VIIRS through the use of infrared absorption bands constructed from VIIRS and CrIS data fusion" by E. Eva Borbas et al.
}

Anonymous Referee \#1

Received and published: 17 August 2020

Review of "Improvement in tropospheric moisture retrievals from VIIRS through the use of infrared absorption bands constructed from VIIRS and CrIS data fusion" manuscript, by E. Borbas et al.

The manuscript presents a novel way to retrieve humidity columns from the spatially highly resolved VIIRS imaging instrument with the hyperspectral sounder CrIS at high spatial sampling and hence enable continuity with the MODIS products. It is found overall well structured and well written, with clear and concise discussions and appropriate choice of illustrations. The study is interesting in particular in the perspective of 
climate studies, to enable continuous records of humidity products originating from different programmes. The manuscript is understood as a demonstration of the potential of the method, which could be further elaborated and validated in a second step. The manuscript is recommended for publication with minor revisions, addressing the few general and specific suggestions below.

Interactive

\section{General:}

comment

The introduction and conclusion should clarify the scope of this paper, i.e. to test a first attempt to aim continuity with MODIS humidity products by attempting retrievals from data fusion. But with further work needed for validation (e.g. longer periods, use of independent reference measurements...) and possibly for the retrieval method itself. It is surprising that IR+MW retrievals (NUCAPS) gives so large dry biases compared to the other products. Because it is the only product exploiting microwave measurements, one would expect good precision here. Can the authors discuss these observations a little bit? In particular drawing from respective validation papers and reports published in the past. Is it envisaged at some stage to perform retrievals from multi-spectral data fusion: VIIRS+CrIS+ATMS? I.e. to try and exploit the maximum information content and enable production in cloudy conditions too. A line or two to comment on this, e.g. in the conclusion/outlook or a brief discussion in what no added-value may be expected would give useful perspective to the reader.

Specific:

P4.L13-14 /Fig.2: clarify if the "mean clear-sky BTDs" is a bias (=mean) or a standard deviation ("increasing measurement noise" of L17). If mean=bias, then what is the actual dispersion, which is the real noise increase.

P5.L8: not the object of this paper, but 15000 training patterns seems rather small in common ML approaches nowadays. Can the authors comment about possible limitations with this approach? 
P8.L16: "also performs well ... but ..." I suggest avoiding to say that a product performs well on the reason that it compares better to MODIS. In particular here, we should expect that the utilisation of microwave together with hyperspectral infrared sounders offers higher capabilities for accurate TPW characterisation than the MODIS imager on its own. Especially in very moist to cloudy atmospheres. Sampling effects of CrIS/ATMS vs MODIS resolution may however explain small dry bias in moist atmospheres and conversely. It would be instructive to compute spatial averaging of MODIS products at CrIS spatial resolution to see if the dry bias on the moistest atmospheres remains.

A comparison to e.g. ground-based TPW sensing (Bedka et al. 2010 or Roman et al. 2016) is advisable to be able to conclude on the final respective skills of the fusion and the single products. It is however an interesting results to match closely MODIS retrievals with the fusion products, which can be highlighted as such.

P13.L12 - P14.L1-2: the link between the former results (2011...) and the present study and the conclusion asserted is not clear. The argument would deserve some elaboration. VIIRS+CrIS TPW could agree very well with MODIS TPW even in the absence of a 3rd reference dataset. However it would be difficult to infer which one is closer to the truth.

P14.L12 and P16/L16: clarify if MODIS UTH is really MYD07 or indeed MYD08 as per manuscript introduction. If MYD07, some more details of that version vs MYD08 would be useful to the readu to interpret the results.

The conclusion should open to validation against fiducial reference measurements to conclude on the differences between MODIS and the fusion products, or explain in what this is judged not necessary, if so.

Interactive comment on Atmos. Meas. Tech. Discuss., doi:10.5194/amt-2020-248, 2020.

Printer-friendly version

Discussion paper
Interactive comment 